\title{
Handover Modeling of Multiple States of Mobile Node in a Five Node Network Model
}

\author{
Suresh R. Halhalli \\ Department of Electronics \\ M.S. Bidve Engineering College \\ Latur (MS), India
}

\author{
Subhash Kulkarni \\ Department of ECE \\ PESIT, South Campus, \\ Bangalore (KS), India
}

\author{
K. S. R. Anjaneyulu \\ Department of EEE \\ JNTUA College of Engineering \\ Anantapuramu (AP), India
}

\begin{abstract}
In this work, handover probability, unnecessary handover probability, missing handover probability and wrong decision probability are computed for a five node network model. The four states of mobile node, namely Cooperative state, failed state, selfish state and malicious state are also modeled. The simulation results are presented for a case of bandwidths up to 20 . Decision time has been varied from $11 \mathrm{~ms}$ to $15 \mathrm{~ms}$. Finally important conclusions are drawn and future work is defined.
\end{abstract}

\section{Keywords}

Wrong Decision Probability, Missing Handovers, Unnecessary handovers, five node network model and multiple states.

\section{INTRODUCTION}

In today's world, user demands keep changing at a faster rate. To keep up the pace with the fast changing needs, technology also needs to change accordingly. The technology and the topology of the wireless networks are designed and operated to suit the user needs. Various parameters of the networks are optimized for usage while designing the networks. These parameters include but not limited to the bandwidth, signal strength, processing speed, voice data transfer, video transfer, movement of the mobile node etc. More importantly, the networks should have the capability to handover the mobile node to another network seamlessly. There are several conditions on which the handover must happen and the networks should have the capability to change the criteria for effective handover under different conditions. However it is difficult for the networks to handover the mobile node seamlessly due to continuously changing conditions at the target network. At the end, the handover turns out to an unnecessary handover or missing handover. Unnecessary hand over happens when the network decides to handover based on certain criteria, but that criteria no more exists when the actual transfer takes place.

There are several algorithms already available based on which the handover happens in the literature [1-3]. Authors presented the handover algorithms based on the bandwidth availability, received signal strength, access delay and rate of displacement of the mobile node. For example, handover happens when the signal strength at the network falls below the threshold value [2]. Similarly handover can happen when the bandwidth falls below the threshold value and the bandwidth at the target network is higher than the threshold value in the target network [3]. Performance of all such algorithms mentioned above is assessed based on the conditions made in assumptions while developing the algorithms. The simulated performance may be quite different from the actual performance. Other algorithms can be found in references [9-19].

The performance of these algorithms which are based on combinations of different decision making parameters are need to be assessed to identify the one which produces best results in terms of successful handover. The performance measuring algorithms should be independent of the technology used in the handover algorithm. The performance can be based on number of successful handovers or number of incorrect decisions. Hence it necessitates a common algorithm to measure the performance. There are some performance measurement algorithms available in [4, 5]. These algorithms do not have robustness in terms of analytical approach and mathematical formulation. However this gap was filled by a new approach [6] based on the probability models and is independent of the technology used for the handover. The approach is known as wrong decision probability and is based on number of incorrect decisions made for hand over. Wrong decision probability approach is based on the criteria like bandwidth availability, signal strength, movement of the mobile node etc. Authors in [6] used a two network model which was academic in nature. However, in practice the number of networks are more than two, many cases it is five. Suresh et. al. developed a five network model to measure the performance based on wrong decision probability [7]. The wrong decision probability was computed based on available bandwidth. Other algorithms that evaluate the performance can be found in [9-21]. In all the algorithms that the authors developed in [7], the mobile nodes were assumed to be in good health. However in practice not all the mobile nodes in the network are cooperative, but can be in other states like failed state, selfish state or malicious state [20, 21]. In this work, a five node network model is developed to consider all the four states, namely, cooperative state, failed state, selfish state and malicious state in calculating the handover probability, unnecessary handover probability, missing handover probability and wrong decision probability based on the criteria of available bandwidth. Next section presents the analytical models for the UHP, HP and WDP. Section III has the general algorithms which are used in calculating the probabilities and section IV presents the simulated results.

\section{ANALYTICAL MODEL}

Consider network model where there are five network nodes. These nodes can be in any of the four possible states, namely, Cooperative, Malicious, Selfish or Failed State. Definitions for these four states can be found in ref. $[20,21]$. The five node network model proposed by [7] has all the nodes in cooperative state and may be referred to as single state model. In this work, three other states are considered where the mobile can lie. The model that is presented in this work may be called as four state model.

\subsection{Single State Model:}

A network having five nodes $n_{1}, n_{2}, n_{3}, n_{4}$, and $n_{5}$ is shown in Fig. 1 and the available bandwidth for the five networks are $B_{1}, B_{2}, B_{3}, B_{4}$, and $B_{5}$ respectively. 
Let $P_{n i / n i}$ : The probability of mobile node continues to stay in $n_{i}$ after a time interval $\mathrm{D} m s$.

$$
P_{n i / n i}=1-\left[\sum_{\substack{j=1 \\ j \neq i}}^{5} P_{n j / n i}\right]
$$

As presented in [7], probability that a mobile node continues to stay in the network node 1 , node 2 , node 3 , node 4 , or node 5 is given below. The five node network model can be represented using Markov five state model [8], and the probabilities that a mobile node stays at $n_{1}, n_{2}, n_{3}, n_{4}$, and $n_{5}$ can be expressed as

$$
P_{n 1}=\frac{P_{n 1 / n 2}+P_{n 1 / n 3}+P_{n 1 / n 4}+P_{n 1 / n 5}}{P_{n 1 / n 2}+P_{n 2 / n 1}+P_{n 1 / n 3}+P_{n 3 / n 1}+P_{n 1 / n 4}+P_{n 4 / n 1}+P_{n 1 / n 5}+P_{n 5 / n 1}}
$$

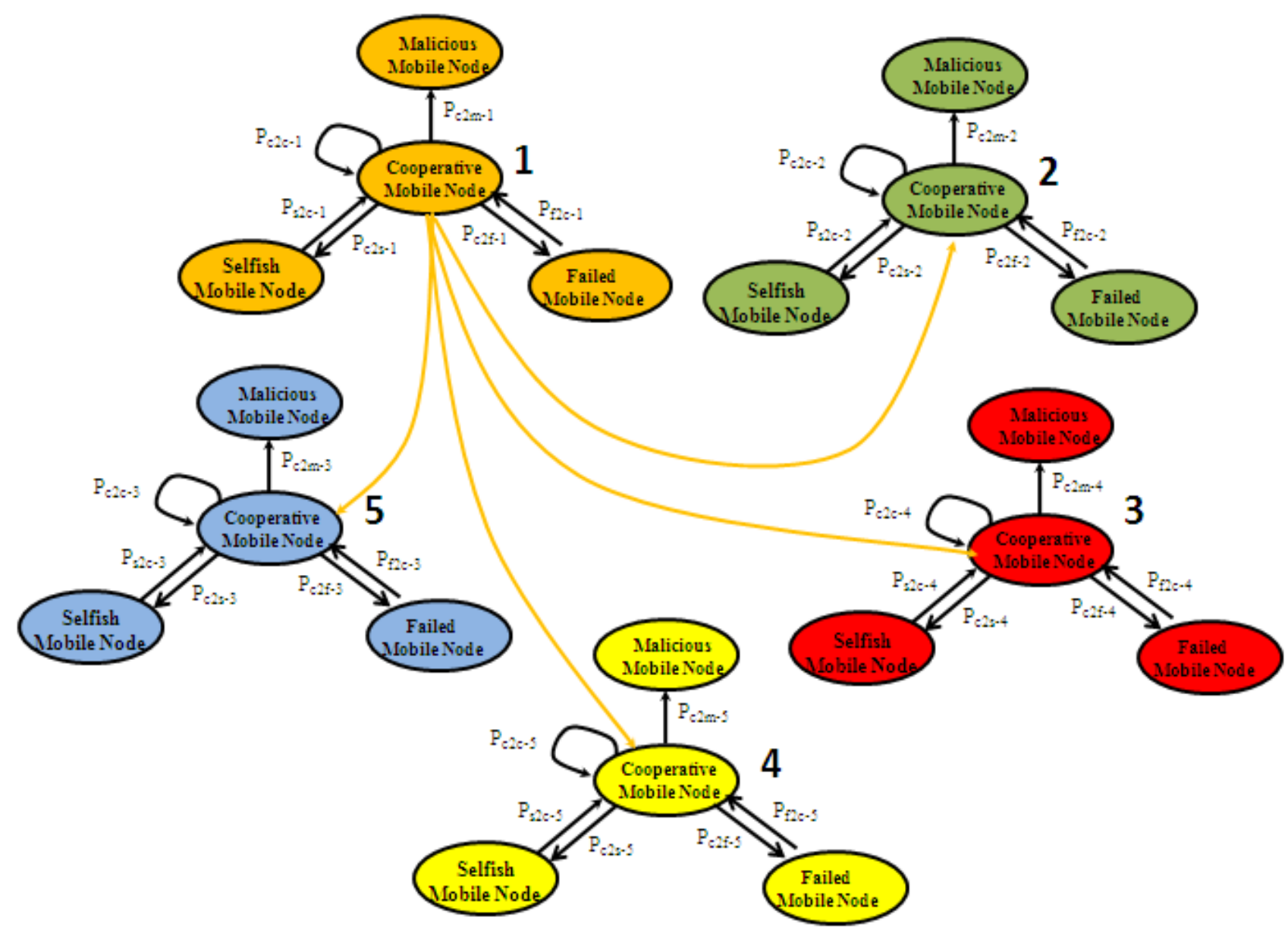

Fig 1: Markov State Five Node Network Model

\subsection{Four State Model:}

Fig. 1 shows the 5 node network model and the states of each mobile node staying in that network. For example, the mobile node in the network can stay in cooperative state, failed state, selfish state or malicious state. The mobile node $\mathrm{m}$ can continue to stay in cooperative state and its probability is shown as $P_{\mathrm{c} 2 \mathrm{c}-\mathrm{m}}$. It can move from cooperative state to failed

$$
P_{n 2}=\frac{P_{n 2 / n 1}+P_{n 2 / n 3}+P_{n 2 / n 4}+P_{n 2 / n 5}}{P_{n 2 / n 1}+P_{n 1 / n 2}+P_{n 2 / n 3}+P_{n 3 / n 2}+P_{n 2 / n 4}+P_{n 4 / n 2}+P_{n 2 / n 5}+P_{n 5 / n 2}}
$$$$
P_{n 3}=\frac{P_{n 3 / n 1}+P_{n 3 / n 2}+P_{n 3 / n 4}+P_{n 3 / n 5}}{P_{n 3 / n 1}+P_{n 1 / n 3}+P_{n 3 / n 2}+P_{n 2 / n 3}+P_{n 3 / n 4}+P_{n 4 / n 3}+P_{n 3 / n 5}+P_{n 5 / n 3}}
$$$$
P_{n 4}=\frac{P_{n 4 / n 1}+P_{n 4 / n 2}+P_{n 4 / n 3}+P_{n 4 / n 5}}{P_{n 4 / n 1}+P_{n 1 / n 4}+P_{n 4 / n 2}+P_{n 2 / n 4}+P_{n 4 / n 3}+P_{n 3 / n 4}+P_{n 4 / n 5}+P_{n 5 / n 4}}
$$

$$
P_{n 5}=\frac{P_{n 5 / n 1}+P_{n 5 / n 2}+P_{n 5 / n 3}+P_{n 5 / n 4}}{P_{n 5 / n 1}+P_{n 1 / n 5}+P_{n 5 / n 2}+P_{n 2 / n 5}+P_{n 5 / n 3}+P_{n 3 / n 5}+P_{n 5 / n 4}+P_{n 4 / n 5}}
$$

Respectively

Where

$P_{n j / n i}:$ The probability of mobile node moving from node $n_{i}$ to $n_{j}$

state or selfish state or malicious state. After it moves to failed state or selfish state, it can return back to cooperative state, but if it goes into malicious state it will not return to cooperative state. It is shown in the Fig. 1; a mobile node that is in cooperative state in network node $n_{1}$ can move to any of the network nodes $n_{2}, n_{3}, n_{4}$, or $n_{5}$ and vice versa is also 
possible. However to minimize the complexity of the Fig. 1 it is not graphically shown here.

The probabilities that a mobile nodes stay in the respective network nodes 1 to 5 and in cooperative state are

$P_{c 2 c-1}=\frac{P_{n 1 / n 2}+P_{n 1 / n 3}+P_{n 1 / n 4}+P_{n 1 / n 5}}{P_{n 1 / n 2}+P_{n 2 / n 1}+P_{n 1 / n 3}+P_{n 3 / n 1}+P_{n 1 / n 4}+P_{n 4 / n 1}+P_{n 1 / n 5}+P_{n 5 / n 1}}$

$P_{c 2 c-2}=\frac{P_{n 2 / n 1}+P_{n 2 / n 3}+P_{n 2 / n 4}+P_{n 2 / n 5}}{P_{n 2 / n 1}+P_{n 1 / n 2}+P_{n 2 / n 3}+P_{n 3 / n 2}+P_{n 2 / n 4}+P_{n 4 / n 2}+P_{n 2 / n 5}+P_{n 5 / n 2}}$

$P_{c 2 c-3}=\frac{P_{n 3 / n 1}+P_{n 3 / n 2}+P_{n 3 / n 4}+P_{n 3 / n 5}}{P_{n 3 / n 1}+P_{n 1 / n 3}+P_{n 3 / n 2}+P_{n 2 / n 3}+P_{n 3 / n 4}+P_{n 4 / n 3}+P_{n 3 / n 5}+P_{n 5 / n 3}}$

$P_{c 2 c-4}=\frac{P_{n 4 / n 1}+P_{n 4 / n 2}+P_{n 4 / n 3}+P_{n 4 / n 5}}{P_{n 4 / n 1}+P_{n 1 / n 4}+P_{n 4 / n 2}+P_{n 2 / n 4}+P_{n 4 / n 3}+P_{n 3 / n 4}+P_{n 4 / n 5}+P_{n 5 / n 4}}$

$P_{c 2 c-5}=\frac{P_{n 5 / n 1}+P_{n 5 / n 2}+P_{n 5 / n 3}+P_{n 5 / n 4}}{P_{n 5 / n 1}+P_{n 1 / n 5}+P_{n 5 / n 2}+P_{n 2 / n 5}+P_{n 5 / n 3}+P_{n 3 / n 5}+P_{n 5 / n 4}+P_{n 4 / n 5}}$

The probabilities that Mobile node continues to stay in cooperative state in the network node 1 , node 2 , node 3 , node 4 , or node 5 are given as

$P_{n 1}=P_{c 2 c-1}+P_{f 2 c-1}+P_{s 2 c-1}-P_{c 2 s-1}-P_{c 2 m-1}-P_{c 2 f-1}$

$P_{n 2}=P_{c 2 c-2}+P_{f 2 c-2}+P_{s 2 c-2}-P_{c 2 s-2}-P_{c 2 m-2}-P_{c 2 f-2}$

$P_{n 3}=P_{c 2 c-3}+P_{f 2 c-3}+P_{s 2 c-3}-P_{c 2 s-3}-P_{c 2 m-3}-P_{c 2 f-3}$

$P_{n 4}=P_{c 2 c-4}+P_{f 2 c-4}+P_{s 2 c-4}-P_{c 2 s-4}-P_{c 2 m-4}-P_{c 2 f-4}$

$P_{n 5}=P_{c 2 c-5}+P_{f 2 c-5}+P_{s 2 c-5}-P_{c 2 s-5}-P_{c 2 m-5}-P_{c 2 f-5}$

As the mobile node can stay in any of the state like, cooperative state, failed state, selfish state or malicious state, it has to move from any of the failed state, selfish state or malicious state into cooperative state for effective functioning. Hence probability of a mobile node present in cooperative state determines the successful handover probabilities as well. The probabilities of a mobile node moving from failed state, selfish state or malicious state into cooperative state are presented in $[20,21]$.

The Probability of a mobile node $m$ moving from failed state to cooperative state can be represented as

$$
P_{f 2 c-m}=\frac{1}{T_{\operatorname{Re} c-m}}
$$

Similarly, Probability of the mobile node $m$ moving from selfish state to cooperative state can be represented as

$$
P_{s 2 c-m}=\frac{T C_{T h r-m}}{T C_{\text {Max }-m}}
$$

Malicious state is a dead state of the mobile node where it does not respond to network signals. Hence the probability of a mobile node moving from malicious state to cooperative state is nil.
Also the probabilities of the mobile node moving from the cooperative state to the any of the failed state, selfish state or malicious state are presented in [20,21].

The probability of the mobile node $m$ moving from cooperative state to selfish state can be represented as

$$
P_{c 2 s-m}=\frac{1}{T_{\text {Selfish }-m}}
$$

Probability of the mobile node moving from cooperative state to malicious state can be represented as

$$
P_{c 2 m-m}=q_{a} \frac{k_{a}}{N} \frac{1}{T_{\text {attack-m }}}
$$

Probability of the mobile node moving from cooperative state to failed state can be represented as

$$
P_{c 2 f-m}=\max \left(\frac{1}{T_{\text {Life-m }}}, \frac{1}{T_{\text {Residence- } m}}\right)
$$

In this work, these probabilities are used to determine the hand over probabilities.

Handover probabilities are given by

$$
H P=\frac{1}{5}\left\{\begin{array}{l}
P_{n 1}\left(P_{n 2 / n 1}+P_{n 3 / n 1}+P_{n 4 / n 1}+P_{n 5 / n 1}\right)+ \\
P_{n 2}\left(P_{n 1 / n 2}+P_{n 3 / n 2}+P_{n 4 / n 2}+P_{n 5 / n 2}\right)+ \\
P_{n 3}\left(P_{n 1 / n 3}+P_{n 2 / n 3}+P_{n 4 / n 3}+P_{n 5 / n 3}\right)+ \\
P_{n 4}\left(P_{n 1 / n 4}+P_{n 2 / n 4}+P_{n 3 / n 4}+P_{n 5 / n 4}\right)+ \\
P_{n 5}\left(P_{n 1 / n 5}+P_{n 2 / n 5}+P_{n 3 / n 5}+P_{n 4 / n 5}\right)
\end{array}\right\}
$$

Wrong decision probability can be computed from the unnecessary handover probability (UHP) and missing handover probability (MHP). The exact expressions for the probabilities can be referred from ref $[6,7]$. The difference between the single state model and four state model lies only in the expressions of the probabilities for $P_{n 1}$ and $P_{n 2}$. Wrong probability is the sum of UHP and MHP as defined in $[6,7]$.

$W D P=U H P+M H P$

\section{GENERAL ALGORITHM}

The general algorithm that is used in this simulation is similar to the one used in the ref [7]. Band width alone is considered as the performance metric for the handover in this algorithm. The details of the algorithm can be found in the ref [7].

\section{RESULTS OF SIMULATION}

Bandwidths are varied from 1 to a maximum of 20 on all the five network nodes and simulation are run on the above model. The model was coded in MATLAB. The decision time is also varied for $\mathrm{D}=11 \mathrm{~ms}$ to $15 \mathrm{~ms}$. Since the decision time is varied, the probabilities of mobile node moving from cooperative state to failed state, selfish state or malicious state and vice versa also varies. In this model following factors are used for simulation.

- $\quad$ Lift Time, $T_{\text {Life-m }} \quad$ = D

- $\quad$ Residence time, $T_{\text {Residence- } m}=\mathrm{D} / 4$ 
- Attack time, $T_{\text {attack-m }}=\mathrm{D} / 4$

- Recovery time, $T_{\operatorname{Re} c-m} \quad=\mathrm{D} / 4$

- Selfish time, $T_{\text {Selfish }-m}=\mathrm{D} / 4$

- Probability of attack, $\mathrm{q}_{\mathrm{a}}=0.3$

- $\frac{T C_{T h r-m}}{T C_{M a x-m}}=0.01$

- $\frac{k_{a}}{N} \quad=0.01$

Fig. 2 shows the handover probabilities versus bandwidth (traffic density) for four state model for different decision times, $\mathrm{D}=11$ to $15 \mathrm{~ms}$. As the handover probabilities depend on the decision time, it can be noticed that the handover probability has inverse replation with the decision time. Handover probabilities are high for $\mathrm{D}=15 \mathrm{~ms}$ compared to $\mathrm{D}=11$ to $14 \mathrm{~ms}$. whereas the handover probabilities are independent of the decision time in case of a single state model.

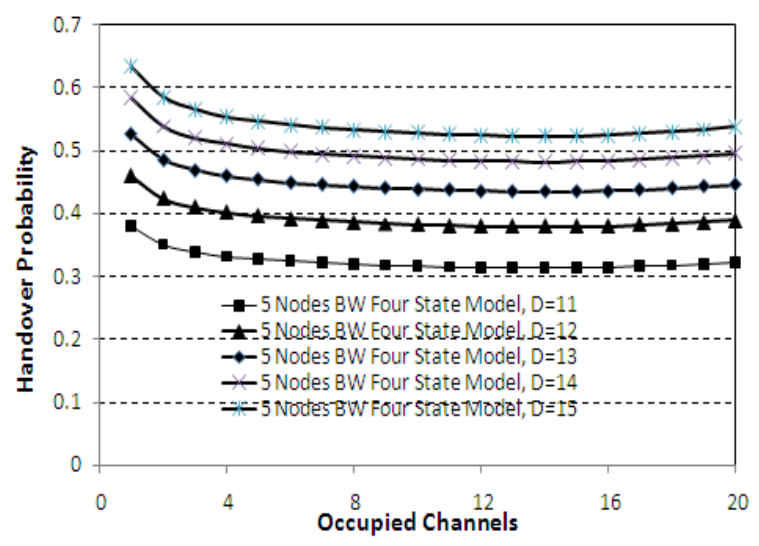

Fig 2: Handover Probability versus Traffic Density for Four State Model for $D=11$ to $15 \mathrm{~ms}$.

The handover probabilities decrease with increase of the traffic density. Fig. 3 shows the Handover Probability versus Traffic Density for Single State and Four State Model for a case of $\mathrm{D}=11 \mathrm{~ms}$.

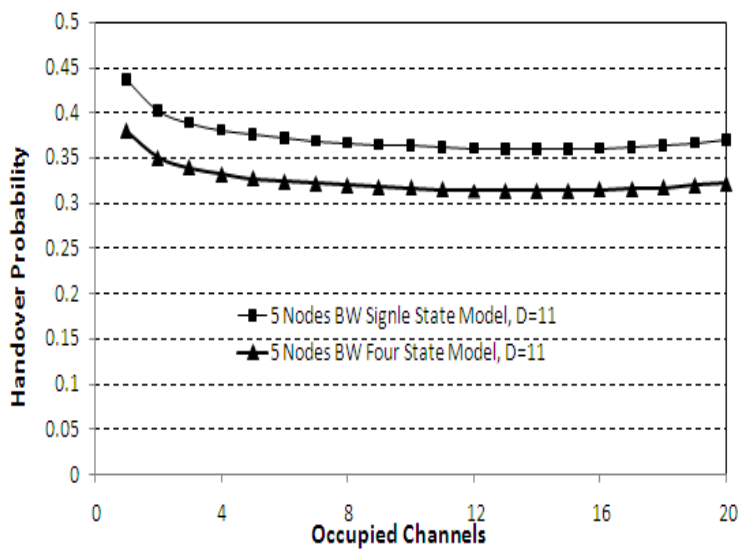

Fig 3: Handover Probability versus Traffic Density for Single State and Four State Models for $D=11 \mathrm{~ms}$.

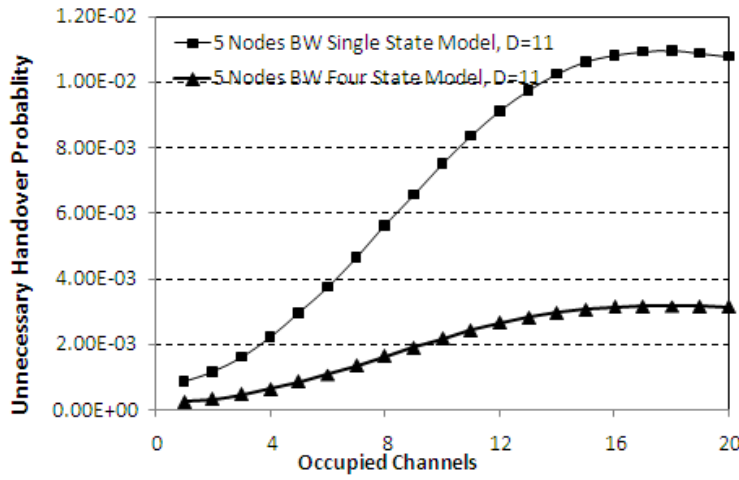

Fig 4: Unnecessary Handover Probability versus Traffic Density for Single State and Four State Models for $D=11$ ms.

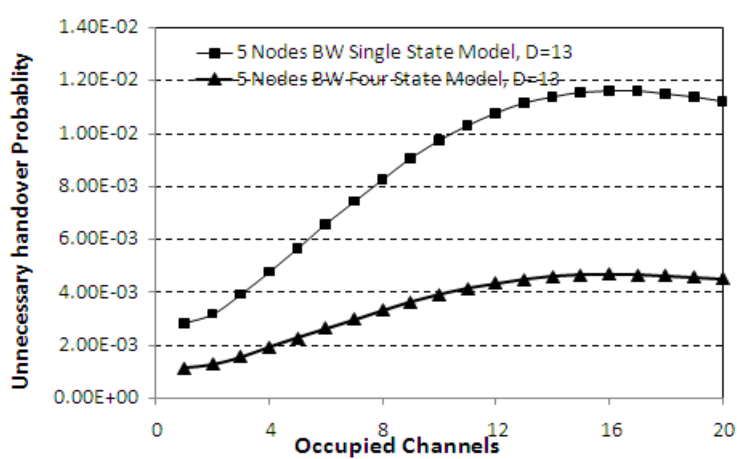

Fig 5: Unnecessary Handover Probability versus Traffic Density for Single State and Four State Models for $D=13$ ms.

Figs. 4 and 5 show the UHP versus traffic density for single state and four state models respectively. It can be noticed that the unnecessary handover probabilities in the four state models is less than half that of the single state models. In the single state models, the model is oversimplified with assumptions like all the nodes are healthy and hence it resulted in higher values of probabilities. The reason behind less probability in case of four state models is that the probablities get modified by the factors of other states like failed state, selfish state and malicious state. For a traffic densify of 16 onwards, the UHP becomes stangnant and in fact it drops down slowly, but not as signficantly as it raised for the case of traffic density upto 16.

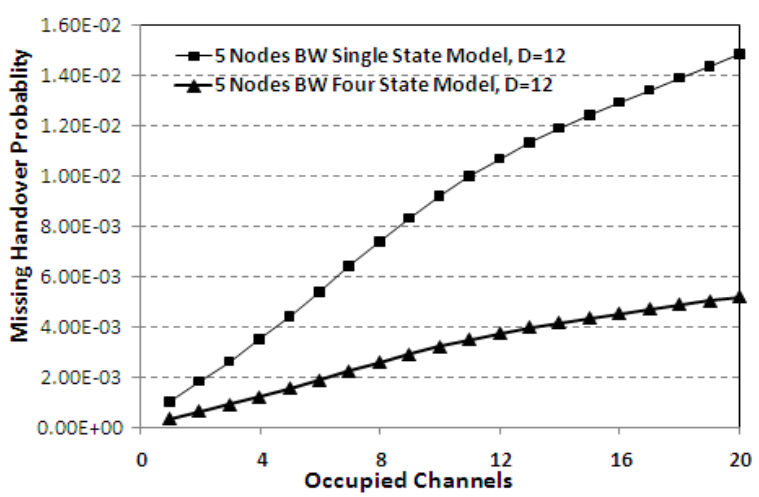

Fig 6: Missing Handover Probability versus Traffic Density for Single State and Four State Models for $D=12$ ms 


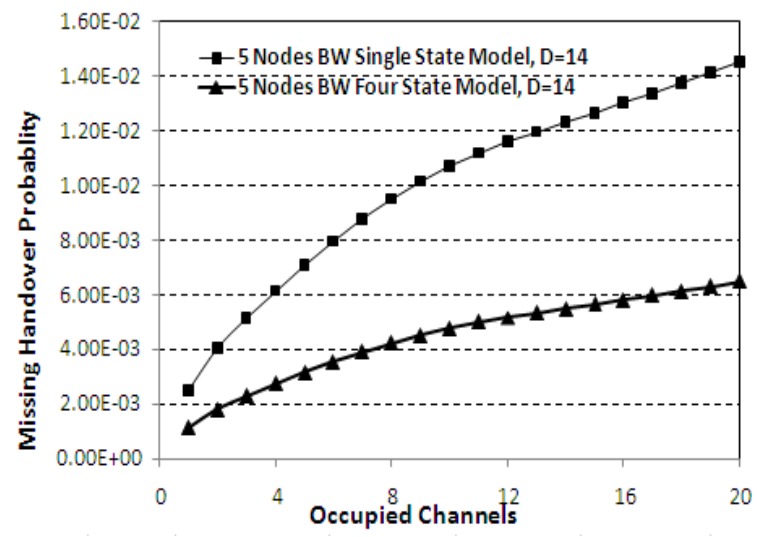

Fig 7: Missing Handover Probability versus Traffic Density for Single State and Four State Models for $D=14$

ms

Figs. 6 and 7 show the MHP versus traffic density for single state and four state models respectively. It can be observed that the missing handover probabilities in the four state model are less by around $70 \%$ than the single state models. Unlike UHP, there is continuous increase in the MHP as the traffic density increases. But the MHP grows at the lesser pace for the four state model than the single state model since the single state model is over simplified as explained above.

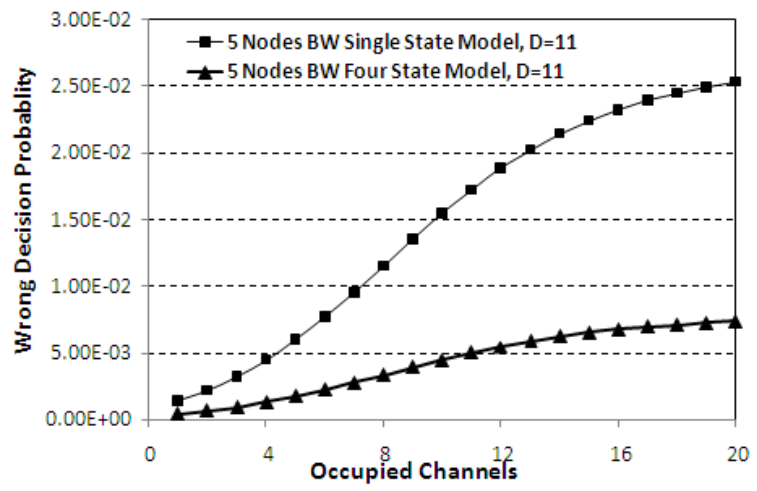

Fig 8: Wrong Decision Probability versus Traffic Density for Single State and Four State Models for $D=11$ ms.

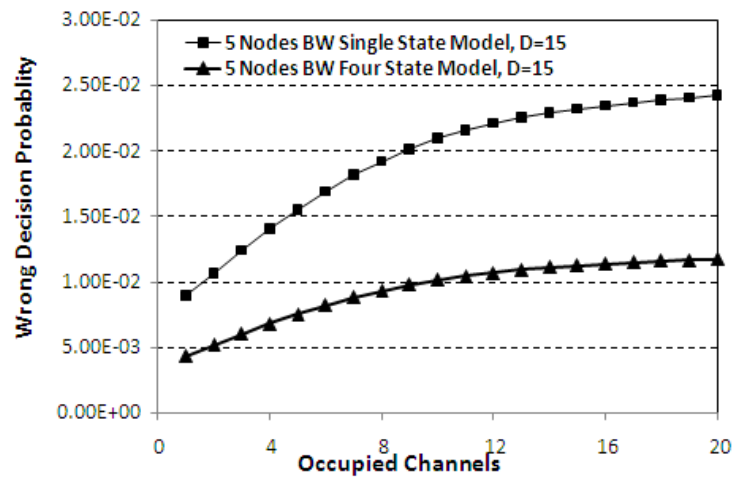

Fig 9: Wrong Decision Probability versus Traffic Density for Proposed Model for $\mathrm{D}=15 \mathrm{~ms}$.

Wrong decision probability (WDP) may be defined as the combined probabliites of the unnecessary and missing handover probabilities, that is, WDP is the summation of the UHP and the MHP. The profiles of the wrong decision probabilities are shown in Figs. 8 and 9. It shows the probabilities of the wrong handovers, because of either unnecessary handover or a missing handover.

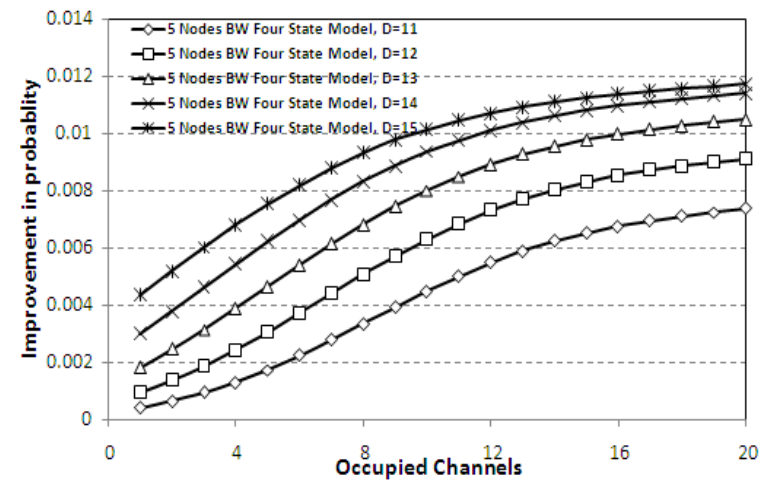

Fig 10: Wrong Decision Probability versus Traffic Density for Single State and Four State Models

Fig. 10 shows the WDP for the cases of $D=11$ to $D=15 \mathrm{~ms}$. The WDP increase as the decision time increases from $11 \mathrm{~ms}$ to $15 \mathrm{~ms}$. This is due to the fact that, more the decision time, more the probability that the conditions in the other network changes. That is, as the decision time increases, the bandwidth on the other node will vary in that duration and the decision is based on the conditions at the beginning of the duration.

\section{CONCLUSION}

In this work, handover probability, Unnecessary Handover Probability, Missing Handover Probability and Wrong Decision Probability are calculated for the bandwidth upto 20 . The decision times are varied from $11 \mathrm{~ms}$ to $15 \mathrm{~ms}$. The models are of two types, namely, single state models and four state models. The single state model is a special case of the four state model. The four states of the model, where the mobile node can exist are coopertaive, failed, selfish and malicious states. If only cooperative state is considered in the model, it is referred to as the single state model. Four state model is very close to the actual sinario and single state model is an over simplified model, which is of only acedemic interest in nature. It is proved with this invetsigation that, the probabilities of the UHP, MHP and WDP of four state models in five node network model are much less than that of the singe stage models. UHP is less by at least $50 \%$ and MHP is less by at least $70 \%$ and so is the case with WDP. The HP depends on the decision time in the case of a four state model in contrast to the single state model. Hence, the four state model represent the actual scenario compared to the single state model. As future work this model can be further extended to models considering the bandwidth and signal strength as the parameters, in order to represent a case close to the actual scenarios.

\section{REFERENCES}

[1] J. McNair, F. Zhu, "Vertical handoffs in fourthgeneration multinetwork environments," IEEE Wireless Communications 11 (3) (2004) 8-15.

[2] A. H. Zahran, B. Liang, and A. Saleh, "Signal threshold adaptation for vertical handoff in heterogeneous wireless networks," Mob. Netw. Appl., vol. 11, no. 4, pp. 625640, 2006.

[3] Chuanxiong Guo, Zihua Guo, Qian Zhang, and Wenwu Zhu, "A Seamless and Proactive End-to-End Mobility Solution for Roaming Across Heterogeneous Wireless Networks", IEEE Journal on Selected Areas in Communications,Vol. 22, No. 5, June 2004, pp.834-848. 
[4] W. Zhao, R. Tafazolli, and B. G. Evans, "Internetwork handover performance analysis in a gsm-satellite integrated mobile communication system," Selected Areas in Communications, IEEE Journal on, vol. 15, no. 8, pp. 1657-1671, 1997.

[5] M. N. Halgamuge, H. Le Vu, K. Rarnamohanarao, and M. Zukerman, "Signal-based evaluation of handoff algorithms," Communications Letters, IEEE, vol. 9, no. 9, pp. 790-792, 2005.

[6] C. Chi, X. Cai, R. Hao and F. Liu "Modeling and Analysis of Handover Algorithms" IEEE GLOBECOM 2007 proceedings.

[7] S. R. Halhalli, S. Kulkarni, K. S. R. Anjaneyulu, S. Akhila, "Probability Modeling of Multi Node Wireless Networks," International Journal of Computer Applications (IJCA) (0975 - 8887) Volume 51- No.1, August 2012.

[8] Daniel Heyman and Mattlew Sobe, Stochastic Models in Operations Research, McGraw-Hill, 1982.

[9] G. Pollini, "Trends in handover design," IEEE Commun. Mag., March, 1996.

[10] K. Pahlavan, P. Krishnamurthy, A. Hatami, M. Ylianttila, J. Makela, R. Pichna, and J. Vallstrom, "Handoff in Hybrid Mobile Data Networks," IEEE Personal Communications, Apr. 2001.

[11] M. Ylianttila, M. Pande, J. Makela, and P. Mahonen, "Optimization scheme for mobile users performing vertical handoffs between IEEE 802.11 and GPRS/EDGE networks," in Proc. IEEE Globecom'01.

[12] Xiang-Yang Li, Peng-Jun Wan, Yu Wang, and Chih-Wei Yi. Fault Tolerant Deployment and Topology Control in Wireless Networks. In Proc. of ACM MobiHoc '03, pages 117-128, Jan. 2003
[13] EURESCOM Project P1013-FIT-MIP, "First steps towards UMTS: Mobile IP services, a European test bed", http://www.eurescom.de/public/projects/P1000series /p1013/ default.asp.

[14] J. McNair, I. Akyildiz, and M. Bender, "An inter-system handoff technique for the IMT-2000 system," in Proc. IEEE infocom 2000.

[15] C. Perkins, Editor, "IP mobility support for IPv4," RFC 3344, http://www.ietf.org/rfc/rfc3344.txt

[16] C. Perkins, et al., "Mobility support in IPv6," IETF draft, http://www.ietf.org/internet-drafts/draft-ietfmobileipipv6-24 .txt, work in progress, June, 2003.

[17] Hao Yang, Haiyun Luo, Fan Ye, Songwu Lu, and Lixia Zhang. Security in Mobile Ad Hoc Networks: Challenges and Solutions. IEEE WirelessCommunications, pages 38 -47, Feb 2004.

[18] A. Valko, "Cellular IP: A New Approach to Internet Host Mobility," Computer and Communication Review, vol. 29, no.1, pp. 50-65, January 1999.

[19] R. Ramjee, et al. "HAWAII: A domain-based approach for supporting mobility in wide-area wireless networks," in Proc. IEEE ICNP, 1999.

[20] F. Xing and W. Wang: Modeling and Analysis of Connectivity in Mobile Ad Hoc Networks with Misbehaving Nodes, IEEE ICC 2006 proceeding, IEEE Communications Society.

[21] Patil Shweta and B.N. Manjunatha Reddy, "Modeling and Analysis of Two Node Network Model with Multiple States in Mobile Networks," International Journal of Computer Applications Technology and Research Volume 3- Issue 1, 52 - 55, 2014 